\begin{tabular}{|c|c|}
\hline Title & Finite size scaling of multifractal wave functions: The metal-insulator transition in two-dimensional symplectic systems \\
\hline Author(s) & Y akubo, K.; Ono, M. \\
\hline Citation & $\begin{array}{l}\text { PHY SICAL REV IEW B, 58(15), 9767-9772 } \\
\text { https://doi.org/10.1103Phy SRevB.58.9767 }\end{array}$ \\
\hline Issue Date & 1998-10-15 \\
\hline Doc URL & http:/hdl .handle.net/2115/5648 \\
\hline Rights & Copyright @ 1998 A merican Physical Society \\
\hline Type & article \\
\hline File Information & PRB58-15.pdf \\
\hline
\end{tabular}

Instructions for use 


\title{
Finite-size scaling of multifractal wave functions: The metal-insulator transition in two-dimensional symplectic systems
}

\author{
K. Yakubo and M. Ono* \\ Department of Applied Physics, Hokkaido University, Sapporo 060, Japan
}

(Received 26 May 1998)

\begin{abstract}
A finite-size scaling analysis of wave functions near the metal-insulator transition (MIT) point has been developed, and applied to the MIT in a two-dimensional disordered electron system in the presence of spinorbit interaction. The present method has the following advantages: (i) Quantities characterizing the critical behavior, such as the critical disorder $W_{c}$ or the localization exponent $\nu$, are multiply calculated from independent scaling analyses of spatial parts with different intensities in wave functions. (ii) These quantities and the multifractality of the critical wave function are determined simultaneously. (iii) It is not necessary to treat many samples with different sizes. (iv) Much computing time is saved, and the scaling analysis can be done up to very large sizes. Using this method, we obtained $W_{c}=5.86 \pm 0.04$ and $\nu=2.41 \pm 0.24$ for a model of a two-dimensional symplectic system. [S0163-1829(98)08039-4]
\end{abstract}

\section{INTRODUCTION}

The metal-insulator transition (MIT) in disordered electron systems is one of the most exciting topics in condensedmatter physics. Although significant effort was made for revealing the critical behavior of the MIT during the last two decades, fundamental problems still remain to be clarified. ${ }^{1}$ In theoretical studies of the MIT, numerical approaches are crucial for understanding this continuous quantum phase transition. In actual calculations, however, the limitation of finite system sizes demands some devices to extract precise information on the MIT. The finite-size scaling analysis is the existing most effective way, which assumes that all critical quantities are scaled only by the localization (or correlation) length. Several schemes based on the finite-size scaling idea have been proposed so far. ${ }^{2-7}$ The most popular scheme was developed by MacKinnon and Kramer. ${ }^{3}$ In this scheme, one prepares a quasi-one-dimensional system with a width $L$ and a length $D(D \gg L)$. It is possible to estimate the localization length $\xi_{L}$ of this system from the Green's function or the conductance. In general, the quantity $\xi_{L} / L$ is a function of $L$ and $W$, where $W$ is an external parameter driving the MIT, such as the strength of disorder or the energy. The scaling hypothesis insists that if $L$ is large enough, this function can be written by a single argument function of $\xi / L$ (the scaling function), where $\xi$ is the localization (or correlation) length of the infinite system, i.e., $\xi=\lim _{L \rightarrow \infty} \xi_{L}$. The value of $\xi$ is obtained in a fitting procedure of the scaling function for many different $L$ and $W$. This technique, however, requires quite a long $D$. If the length $D$ is not sufficient, the energy at which the Green's function or the conductance $G_{L}$ is defined has a finite width. This means that several states whose energies are contained in this width contribute $G_{L}$. As a consequence, we tend to evaluate $\xi_{L}$ and $\xi$ as large compared with their true values. The length $D$ for an accurate calculation is extremely long even for small $L$, and increases as $L$ is increased. A long computing time due to the long $D$ prevents us from treating large $L$. For example, $L$ is usually less than $100 a$ for two-dimensional systems, where $a$ is a lattice con- stant. Furthermore, since the system size $L$ only plays the role of the scaling measure, it is necessary to calculate $G_{L}$ for many systems with different sizes.

In a finite-size scaling analysis of quasi-one-dimensional systems, all critical properties are presented by the response function $G_{L}$. Rich information involved in the amplitude distribution of a wave function is discarded. This is so with other finite-size scaling schemes, ${ }^{2,4-7}$ such as the size scaling of level statistics, ${ }^{4-6}$ for instance, which in recent years has been used extensively to determine the nature of the MIT from a sequence of eigenvalues, not from eigenfunctions. In this paper, we present a finite-size scaling method that extracts information on the critical behavior from amplitude distributions of wave functions. This technique is based on the idea that amplitudes of the critical wave function have a multifractal distribution, ${ }^{8,9}$ and even not at the critical point but in the critical region the amplitude distribution is also multifractal in a scale less than $\xi$. As explained in Sec. II, in the present technique a small amount of samples with different sizes is enough for accurate calculations. This superiority enables us to analyze systems up to large sizes. In our method, the amplitude distribution is characterized by the $q$ th moment of squared amplitudes of a wave function. Each scaling analysis for a fixed value of $q$ gives information on the MIT. Since changing the value of $q$ corresponds to analyzing a different part of the wave function, and the critical exponent and the critical value of the external parameter $W$ are independent of $q$, these quantities can be multiply evaluated by alternating $q$.

Using this finite-size scaling analysis, we have determined the critical exponent and the critical disorder of twodimensional symplectic systems. It is widely accepted that the critical behavior at the MIT is cast into three different universality classes by their fundamental symmetries. ${ }^{1,10,11}$ When systems have time-reversal and spin-rotational symmetries, the universal characters of the transition are the same as those of the usual Anderson transition, and an orthogonal class of the MIT is observed. If time-reversal symmetry is broken by a magnetic field, the MIT belongs to the 
unitary class. The third symplectic class is that in which systems do not have a spin-rotational symmetry but a timereversal one, which is realized by spin-orbit interaction in actual systems. In two-dimensional noninteracting electrons, it is believed that only the symplectic system exhibits the MIT. Although recent experimental findings of metallic phases in two-dimensional electron/hole systems at $B=0$ in $\mathrm{Si}$ metal-oxide field-effect transistors $^{12}$ or $\mathrm{GaAs}$ heterostructures ${ }^{13,14}$ encourage one to study the MIT in symplectic systems, a fundamental understanding of the MIT of this class is still insufficient. This is because numerical models of symplectic systems have not appeared until comparatively recently. It is quite important to obtain precise information on the MIT in symplectic systems by applying the present scaling technique.

This paper is organized as follows. In Sec. II, we introduce our finite-size scaling method. Asymptotic behaviors of the scaling function are also discussed in this section. In Sec. III, the nature of the MIT in two-dimensional disordered electrons with spin-orbit interaction are studied by applying our method. The localization length exponent $\nu$ and the critical disorder $W_{c}$ are calculated by analyzing wave functions in very large systems. The mass exponent which characterizes the multifractality of the critical wave function has been also obtained. Conclusions are given in Sec. IV.

\section{FINITE-SIZE SCALING ANALYSIS}

In the newly developed finite-size scaling analysis, we extract information on the critical behavior of the metalinsulator transition (MIT) from amplitude distributions of wave functions. This scaling analysis is based on the fact that the spatial distribution of a squared wave function is multifractal, which gives rise to a spectrum of critical exponents at the critical point of the MIT. ${ }^{8,9}$ Although it is impossible to realize either an infinite system or a critical wave function at the true critical point in numerical calculations, many works have numerically confirmed the multifractality of wave functions near the critical point in finite systems. ${ }^{15}$ This implies that the multifractality of a wave function is not even valid at the critical point if the length scale is less than a characteristic length. According to the one-parameter scaling theory of the MIT, this scale should be the correlation (or localization) length $\xi$. In fact, Fal'ko and Efetov ${ }^{16}$ showed that localized wave functions are multifractal if the system size is smaller than the localization length. It is plausible that a wave function which is localized with a localization length $\xi$, or extended with a correlation length $\xi$, is multifractal on a scale much smaller than $\xi$. If the system is in the critical region, the spectrum of critical exponents describing the multifractality of the wave function coincides with that of the critical wave function. This speculation is supported by an analogy to usual critical phenomena. In percolation problems, for example, the infinite cluster at the percolation threshold has a fractal structure. ${ }^{17}$ The same fractality can be attributed to finite clusters not at the threshold within a length scale less than the correlation length. Our method extracts information on the critical behavior by analyzing how the multifractality of wave functions in the vicinity of the critical point changes with length scales.

In order to characterize the amplitude distribution of a wave function, we introduce a quantity $Z_{q}$ defined by

$$
Z_{q}=\sum_{\operatorname{box}(l)}\left(\sum_{i \in \operatorname{box}(l)}\left|\psi_{i}\right|^{2}\right)^{q},
$$

where $\psi_{i}$ is the amplitude of the wave function at the site $i$, and is normalized as $\Sigma_{i}\left|\psi_{i}\right|^{2}=1$ (the summation is taken over all sites in the system). The symbol $\Sigma_{\text {box }(l)}$ represents the summation over small boxes with a linear size $l$ into which one divides the whole system. The second summation in Eq. (1) is taken over sites in the box. The quantity $q$ is an arbitrary constant. It is well known that if the quantity $Z_{q}$ is proportional to $(l / L)^{\tau(q)}$, where $L$ is the system size and $\tau(q)$ is a nonlinear function of $q$, amplitudes of the wave function have a multifractal distribution. ${ }^{18}$ The $q$ th-order moment of distributed amplitudes $Z_{q}$ depends on $L, l$, and external parameters such as the strength of the disorder $W$ or the energy $E$. If we apply the scaling hypothesis to the amplitude distribution of the wave function near the critical point, the quantity $Z_{q}$ can be written as

$$
Z_{q}=\xi^{\alpha} f_{q}(L / \xi, l / \xi)
$$

where $\alpha$ is an exponent, $\xi$ is the correlation (or localization) length of the infinite system, and $f_{q}$ is a two-argument scaling function which depends on $q$. Since the length $L$ is always larger than $l$, the scaling function $f_{q}(x, y)$ is defined in the regime of $x \geqslant y$.

Let us consider asymptotic forms of the scaling function. At first, we concentrate on the scaling function for localized states. If the localization length $\xi$ is smaller than both the system size $L$ and the box size $l$, i.e., $x \gg 1$ and $y \gg 1$, a box only contributes the quantity $Z_{q}$. Therefore, $Z_{q}$ does not depend on $L$ and $l, \alpha=0$, and $f_{q}(x, y)$ is a constant. From the normalization condition, this constant should be unity. For $x \gg 1$ but $y \ll 1, Z_{q}$ does not depend on $L$, because the wave function is localized inside of the system. The distribution of amplitudes is, however, multifractal within a scale much less than the localization length $\xi$. This means that $Z_{q}$ is proportional to $(l / \xi)^{\tau(q)}$ and $f_{q}(x, y) \propto y^{\tau(q)}$. If $x$ and $y$ are small enough compared with unity, the whole wave function in the system is multifractal. The asymptotic form of the scaling function $f_{q}$ is proportional to $(y / x)^{\tau(q)}$ in this limit. For extended states, we will have a different scaling function than that for localized states. If $x \gg 1$ and $y \gg 1$, one can replace $\left|\psi_{i}\right|^{2}$ in Eq. (1) with an averaged value $\overline{\psi^{2}}$, because the correlation length of the extended state is much smaller than the box size. The quantity $Z_{q}$ is written as $Z_{q} \propto(L / l)^{d}\left(l^{d} \overline{\psi^{2}}\right)^{q}$ in this case. The normalization condition leads to $\overline{\psi^{2}} \propto L^{-d}$. Thus the scaling function in this regime is given by $f_{q}(x, y) \propto(y / x)^{d(q-1)}$. When $x \gg 1$ but $y \ll 1$, the whole wave function can be divided into $(L / \xi)^{d}$ equivalent parts $(\xi$ boxes) whose linear size is $\xi$. Since quantity $Z_{q}$ receives equal contributions from these $\xi$ boxes, $Z_{q} \propto(L / \xi)^{d} z$, where $z$ is the $q$ th-order moment of the amplitude distribution in a $\xi$ box, i.e., $z$ is defined by Eq. (1) replacing the first summation by the summation over small boxes with the size $l$ contained in the $\xi$ box. The quantity $z$ is calculated as follows. We introduce a rescaled wave function $\psi_{i}^{\prime}=r \psi_{i}$ which is normalized within the $\xi$ box. Since all $\xi$ boxes are equivalent, $|r|^{2}=(L / \xi)^{d}$. On a scale less than the correlation length $\xi$, 
the distribution of $\left|\psi_{i}^{\prime}\right|^{2}$ is multifractal. Thus the moment $z^{\prime}$ of $\left|\psi_{i}^{\prime}\right|^{2}$, which is defined by the same definition as that of $z$, except for summing up $\left|\psi_{i}^{\prime}\right|^{2}$ instead of $\left|\psi_{i}\right|^{2}$, becomes $z^{\prime}$ $\propto(l / \xi)^{\tau(q)}$. Substituting $\left|\psi_{i}^{\prime}\right|^{2} \propto(L / \xi)^{d}\left|\psi_{i}\right|^{2}$ into the definition of $z^{\prime}$, one obtains $z^{\prime} \propto(L / \xi)^{d q} z$. Therefore, $Z_{q}$ $\propto(L / \xi)^{d(1-q)}(l / \xi)^{\tau(q)}$ and $f_{q}(x, y) \propto x^{d(1-q)} y^{\tau(q)}$. Finally, in the case of $x \ll 1$ and $y \ll 1$, the wave function cannot be distinguished from the localized one whose localization length is much larger than the system size. The asymptotic form of the scaling function in this limit is the same as that for localized states for $x \ll 1$ and $y \ll 1$. In conclusion, we will have the following asymptotic forms of the scaling function $f_{q}(x, y)$. For localized states,

$$
f_{q}(x, y) \propto \begin{cases}1 & \text { if } x \gg 1 \text { and } y \gg 1 \\ y^{\tau(q)} & \text { if } x \gg 1 \text { and } y \ll 1 \\ \left(\frac{y}{x}\right)^{\tau(q)} & \text { if } x \ll 1 \text { and } y \ll 1,\end{cases}
$$

and, for extended states,

$$
f_{q}(x, y) \propto \begin{cases}\left(\frac{y}{x}\right)^{d(q-1)} & \text { if } x \gg 1 \text { and } y \gg 1 \\ x^{d(1-q)} y^{\tau(q)} & \text { if } x \gg 1 \text { and } y \ll 1 \\ \left(\frac{y}{x}\right)^{\tau(q)} & \text { if } x \ll 1 \text { and } y \ll 1 .\end{cases}
$$

It should be noted that a scaling analysis of the inverse participation ratio ${ }^{19}$ is included as a special case of our analysis. The inverse participation ratio defined by $P^{-1}=\Sigma_{i}\left|\psi_{i}\right|^{4}$ is coincident with $Z_{q}$ for $q=2$ and $l=a$, where $a$ is a lattice constant. Therefore, $l / \xi$ is always less than unity in the $P^{-1}$ analysis. From Eqs. (3) and (4), the asymptotic forms of a scaling function $g(x)$ describing the system size dependence of $P^{-1}$ are expected as follows. For localized states, $g(x)$ $=$ const for $x \gg 1$ and $g(x) \propto x^{-\tau(2)}$ for $x \ll 1$. For extended states, $g(x) \propto x^{-d}$ for $x \gg 1$ and $g(x) \propto x^{-\tau(2)}$ for $x \ll 1$. In this analysis, only system sizes are the scaling measure, and thus it is necessary to prepare many samples with different sizes.

The quantity $Z_{q}$ is calculated from an eigenfunction of a disordered electron system. From the scaling analysis of $Z_{q}$, one obtains the critical disorder $W_{c}$ (or critical energy $E_{c}$ ), the localization length exponent $\nu$, and the mass exponent $\tau(q)$ at the same time. This is one of the prominent features of our method, while exponents $\nu$ and $\tau(q)$ have been calculated separately in previous analyses. In particular, we can calculate $W_{c}$ and $\nu$ multiply for different $q$ 's, because these quantities do not depend on $q$. The scaling analysis for a fixed value of $q$ implies that a part of the amplitudes with a fixed intensity is analyzed. For large $q$, a part of large amplitudes is analyzed, and an analysis for a small-amplitude part is done by choosing a small or negative value of $q$. Since amplitude distributions of these parts with different intensities are independent, ${ }^{18}$ evaluations of $W_{c}$ or $\nu$ by different $q$ 's are independent. Averaging these values, one can determine the precise values of the critical disorder and the critical exponent.

In this method, the scaling measure is changed by the size $l$ of small boxes in addition to the size $L$ of the whole system.
This means that we do not need to calculate eigenstates of many samples with different sizes. In fact, as mentioned in Sec. III, we have treated only two sizes of systems in actual numerical investigation of the MIT in a two-dimensional disordered electron system in the presence of spin-orbit interaction. This feature contrasts with previous methods in which the scaling is done only by the system size $L$. Due to this advantage, the present method enables us to save much computing time and allows us to treat larger systems. Using recent diagonalization techniques and an advanced parallel computer facility, in principle, the finite-size scaling up to $10^{7}$-site systems would be possible.

\section{DELOCALIZATION TRANSITION IN TWO-DIMENSIONAL SYMPLECTIC SYSTEMS}

The scaling theory of noninteracting disordered electrons without spin-orbit interaction predicts that all states are localized in two dimensions. If the spin-orbit interaction becomes strong, the quantum interference acts upon an antilocalization effect. ${ }^{10}$ It is widely accepted that the metalinsulator transition exists in a two-dimensional electron system in the presence of spin-orbit interaction. Since the structure of the Hamiltonian describing this system is invariant under a symplectic transformation, the set of Hamiltonians with this symmetry is called the symplectic ensemble. Disordered electron systems with symplectic symmetry are the only systems which exhibit the MIT in two-dimensional noninteracting electrons. We apply the present scaling technique to the MIT in two-dimensional disordered electrons with spin-orbit interaction.

Several models have been proposed for numerical investigations of the MIT in two-dimensional symplectic systems. ${ }^{20-23}$ Here we employed Ando's model, ${ }^{22}$ that has been studied most extensively. The Hamiltonian on a square lattice is given by

$$
H=\sum_{i, \sigma} \varepsilon_{i}|i, \sigma\rangle\left\langle i, \sigma\left|+\sum_{i, \sigma ; j, \sigma^{\prime}} V\left(i, \sigma ; j, \sigma^{\prime}\right)\right| i, \sigma\right\rangle\left\langle j, \sigma^{\prime}\right|,
$$

where $\varepsilon_{i}$ is a on-site random potential at the site $i$ and distributes uniformly between $-W / 2$ and $W / 2$. The index $\sigma$ $=\uparrow$ or $\downarrow$ denotes the spin-up or -down. If we represent spin states $\langle\uparrow|$ and $\langle\downarrow|$ in $\langle i, \sigma|=\langle i| \otimes\langle\sigma|$ as $(1,0)$ and $(0,1)$, respectively, the matrix forms of the hopping term are

$$
V(i, i+\hat{\boldsymbol{x}})=\left(\begin{array}{cc}
V_{1} & V_{2} \\
-V_{2} & V_{1}
\end{array}\right)
$$

and

$$
V(i, i+\hat{\boldsymbol{y}})=\left(\begin{array}{cc}
V_{1} & -i V_{2} \\
-i V_{2} & V_{1}
\end{array}\right) .
$$

Here $\hat{\boldsymbol{x}}$ and $\hat{\boldsymbol{y}}$ represent the unit vectors of the $x$ and $y$ directions. The strength of the spin-orbit interaction is characterized by $V_{2} / V$, with $V=\left(V_{1}^{2}+V_{2}^{2}\right)^{1 / 2}$. We have chosen this parameter as $V_{2} / V=0.5$. All energies and length scales are measured in units of $V$ and the lattice spacing $a$, respectively. Periodic boundary conditions are applied in both di- 

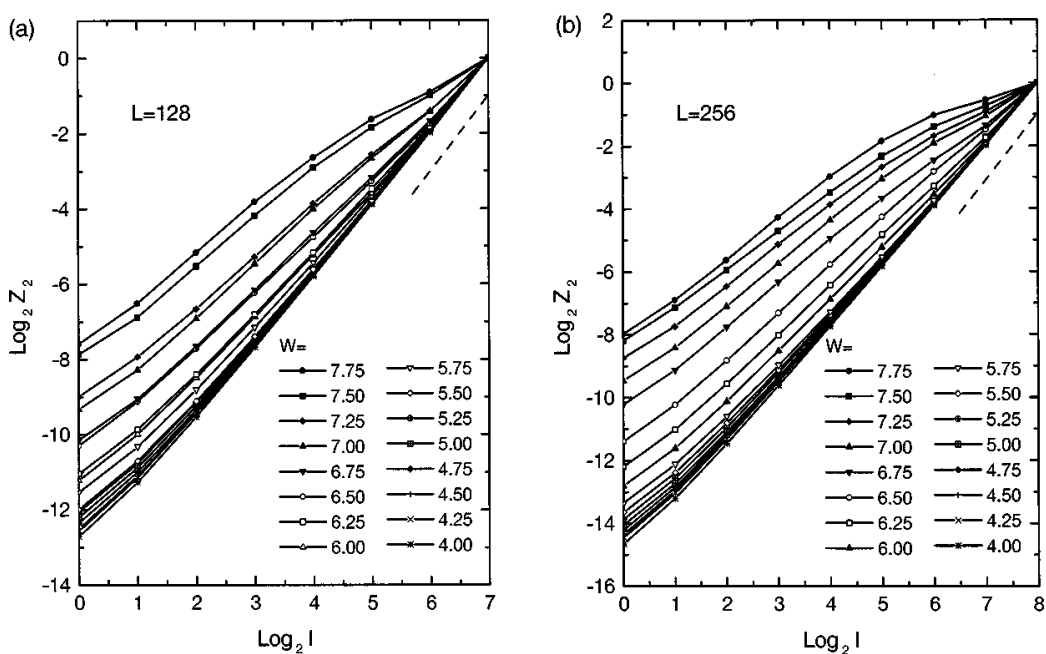

FIG. 1. Box-size dependences of quantities $Z_{q}$ for $q=2$. Marks correspond to different strengths of disorder. Each point has been obtained by averaging $Z_{2}$ over ten realizations of disordered potentials. (a) and (b) represent results for systems with $L=128$ and 256 , respectively.

rections. We concentrate on the band center energy $E=0$, and search the critical disorder $W_{c}$ above which all energy states are localized.

In order to calculate eigenstates of the Hamiltonian [Eq. (5)], we used the forced oscillator method. ${ }^{24}$ This numerical algorithm makes it possible to compute eigenvalues and eigenvectors of very large matrices. In the present work only two sizes ( $L=128$ and 256) of systems have been treated, because in our scaling analysis box sizes play a role of the scaling measure in addition to system sizes. Figure 1 indicates the box size dependence of the quantity $Z_{q}$ for $q=2$. Results for $L=128$ and 256 are shown in Figs. 1(a) and 1(b), respectively. Different marks correspond to different strengths of disorder. Each data point has been obtained by averaging $Z_{2}$ over ten realizations of disordered potentials. Profiles of these lines are consistent with the scaling argument. For strong disorder (large $W$ ), we expect that the eigenstate is strongly localized whose localization length $\xi$ is much smaller than the system size. If the box size $l$ is large compared with $\xi, Z_{2}$ does not depend on $l$. Thus the $l$ dependence of $Z_{2}$ is weak in this regime. When $l$ becomes smaller than $\xi, Z_{2}$ seems to be proportional to $l^{\tau(2)}$, because the wave function is multifractal on this length scale. For weak disorder (small $W$ ), we find concave curves $Z_{2}(l)$. In each line, $Z_{2}$ is proportional to $l^{2}$ when $l$ is large. Dashed lines in Fig. 1 show $Z_{2} \propto l^{2}$. This implies that amplitudes of the wave function distribute almost uniformly on a scale of $l$ much larger than $\xi$. The slope of the line decreases for small $l$, which indicates the multifractal character of the wave function on this scale. The line shape is almost straight for $W \sim 5.75$. This intimates that the critical disorder $W_{c}$ is close to this value.

The scaling functions are calculated from obtained data of $Z_{q}(W, L, l)$ by a fitting procedure. To this end the scaling function $f_{q}(L / \xi, l / \xi)$ is expanded as $f_{q}(x, y)$ $=\Sigma_{m, n} a_{m n} x^{m / \nu} y^{n / \nu}$. We expand this function up to the fifth (a)

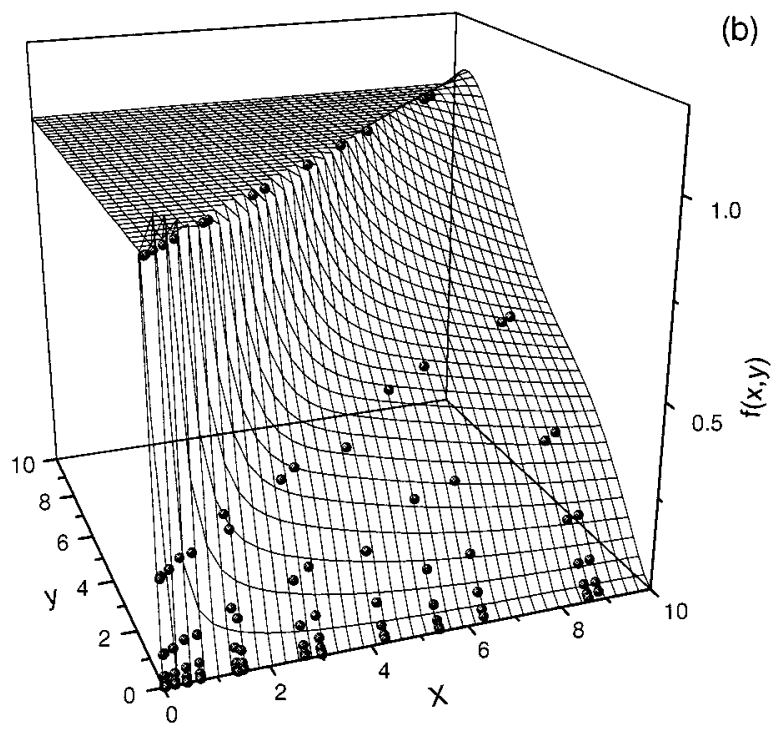

(b)

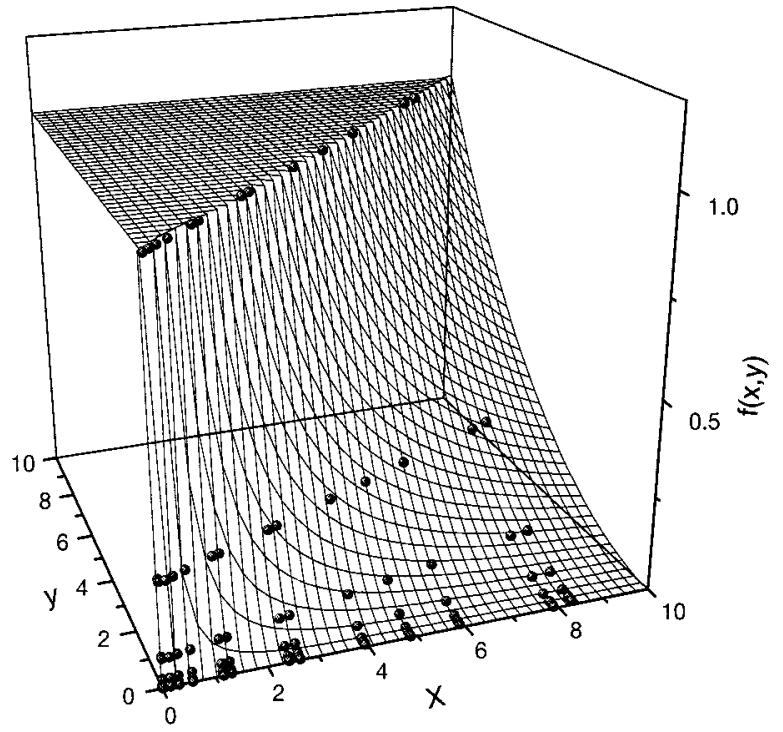

FIG. 2. Two-argument scaling functions $(q=2)$ for (a) localized and (b) extended states. Flat surfaces in the regime $x<y$ have no meaning because the domain of $f_{q}(x, y)$ is $x \geqslant y$. Calculated $Z_{2}$ in Fig. 1 are also plotted in these figures. 

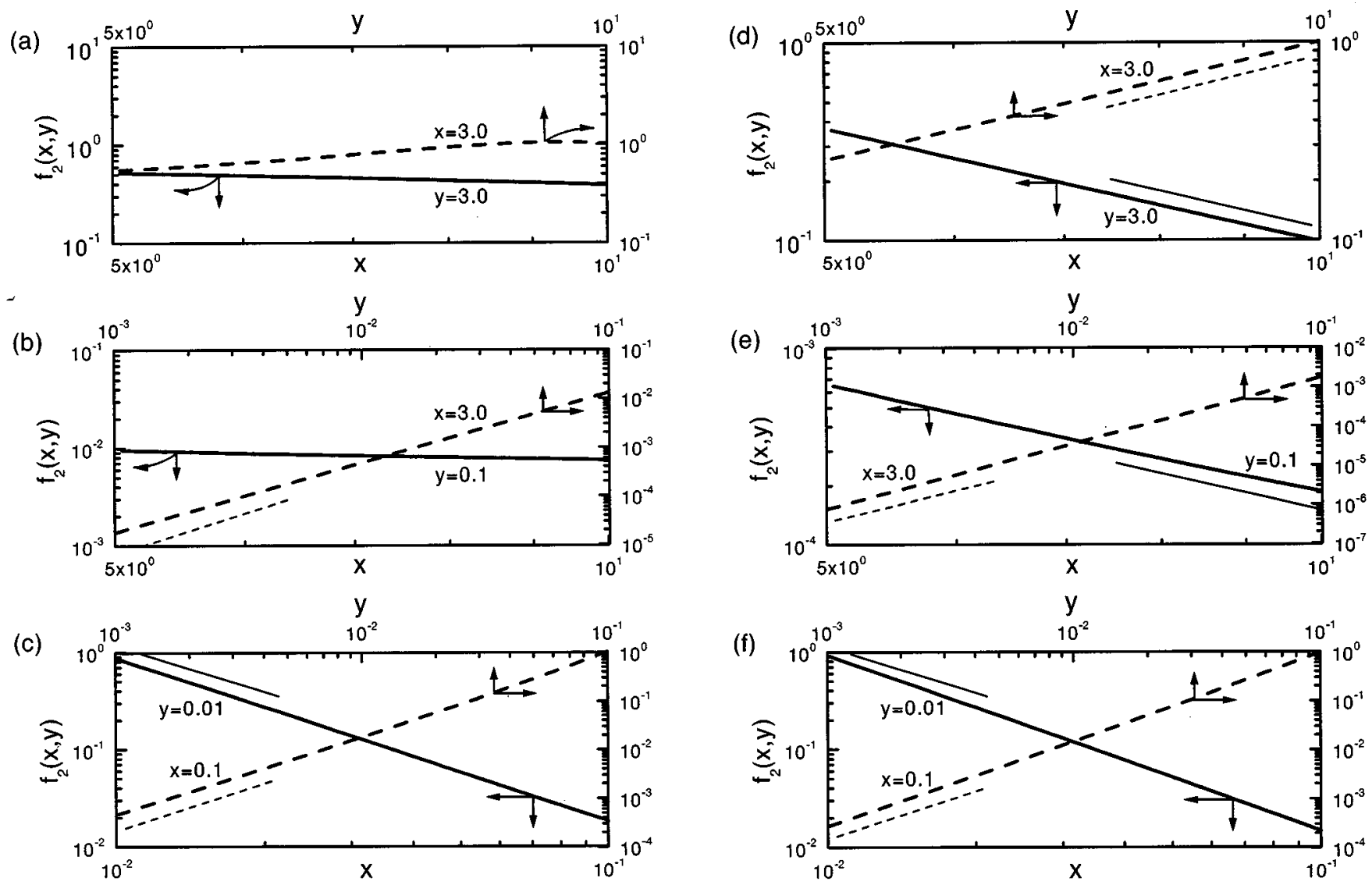

FIG. 3. Asymptotic behaviors of the calculated scaling functions. Solid and dashed lines represent the $x$ and $y$ dependences of the scaling functions for fixed values of $y$ and $x$ (indicated near lines), respectively. (a) The scaling function for localized states in the regime of $x$ $\gg 1$ and $y \gg 1$. (b) The scaling function for localized states in the regime of $x \gg 1$ and $y \ll 1$. Thin dashed line indicates the slope of $f_{2}$ $\propto y^{\tau(2)}$ with $\tau(2)=1.62$. (c) The scaling function for localized states in the regime of $x \ll 1$ and $y \ll 1$. Thin solid and thin dashed lines indicate slopes of $f_{2} \propto x^{-\tau(2)}$ and $f_{2} \propto y^{\tau(2)}$ with $\tau(2)=1.62$, respectively. (d) The scaling function for extended states in the regime of $x$ $\gg 1$ and $y \gg 1$. Thin solid and thin dashed lines indicate slopes of $f_{2} \propto x^{d(1-q)}$ and $f_{2} \propto y^{d(q-1)}$ with $d=2$ and $q=2$, respectively. (e) The scaling function for extended states in the regime of $x \gg 1$ and $y \ll 1$. Thin solid and thin dashed lines indicate slopes of $f_{2} \propto x^{d(1-q)}$ and $f_{2} \propto y^{\tau(2)}$ with $d=2, q=2$, and $\tau(2)=1.62$, respectively. (f) The scaling function for extended states in the regime of $x \ll 1$ and $y \ll 1$. Thin solid and thin dashed lines indicate slopes of $f_{2} \propto x^{-\tau(2)}$ and $f_{2} \propto y^{\tau(2)}$ with $\tau(2)=1.62$, respectively.

order $(m+n \leqslant 5)$. Calculated scaling functions $(q=2)$ for localized and extended states are shown in Figs. 2(a) and 2(b), respectively. Since box sizes are always smaller than the system size, the domain of $f_{q}(x, y)$ is $x \geqslant y$. Flat surfaces for $x<y$ in these figures have no meaning. It should be remarked that $f_{q}(x, x)$ is always unity because of the normalization condition of wave functions. All calculated data of $Z_{2}$ are well embedded onto these scaling surfaces. As shown in Fig. 3, asymptotic behaviors of the calculated scaling functions are consistent with those predicted in Eqs. (3) and (4). In these figures, solid and dashed lines represent the $x$ and $y$ dependences of the scaling functions for fixed values of $y$ and $x$, respectively. Figures 3(a) to 3(c) show profiles of $f_{2}(x, y)$ for localized states in three asymptotic regimes, and Figs. 3(d) $-3(\mathrm{f})$ those for extended states. We find the coincidence between the scaling predictions and numerical results by comparing thick lines (numerical results) with thin lines (scaling predictions).

From the fitting procedure we obtained $W_{c}=5.86 \pm 0.04$, $\nu=2.41 \pm 0.24$, and $\tau(2)=1.62 \pm 0.01$. These values and their statistical errors have been evaluated by using the boot- strap procedure. ${ }^{25,26}$ We have also calculated $W_{c}$ and $\nu$ for several values of $q$ other than $q=2$. Differences between these quantities by varying $q$ from 1.5 to 2.5 are much smaller than their errors. The obtained value of the critical disorder is close to previous numerical results. ${ }^{22,23,27-30}$ The value of $\nu$ presented here is between two previous results $\nu$ $=2.05$ (Ref. 22) and 2.75 (Ref. 27), for which scaling analyses of quasi-one-dimensional systems have been applied to the same model as that in the present work. Our result of $\nu$ is rather close to the value $\nu=2.32$ (Ref. 30) obtained by a scaling analysis of level statistics. The exponent $\tau(2)$ coincides with the generalized dimension of the wave function $D(q)=\tau(q) /(q-1)$ for $q=2$. Kawarabayashi and Ohtsuki ${ }^{29}$ calculated the dimension $D(2)$ numerically from the diffusion of electrons in the same system with this work. Their result is $D(2)=1.68$, and agrees quite well with our $\tau(2)$.

\section{CONCLUSIONS}

We have developed a finite-size scaling method that extracts rich information about critical behavior of the metal- 
insulator transition from amplitude distributions of wave functions, and applied the method to two-dimensional disordered electron systems in the presence of spin-orbit interaction. In contrast to previous scaling analyses in which only the system size is the scaling measure, both the system and box sizes are used for alternating the length scale. Due to this peculiarity it is not necessary to diagonalize Hamiltonians of many systems with different sizes. In this method, the amplitude distribution of a wave function is characterized by its moment of the $q$ th order. By varying the value of $q$, multiple and independent analyses can be performed for a single set of wave functions. It is possible to obtain precise information on the metal-insulator transition from such multiple analyses. Applying the present technique to two-dimensional symplectic systems, we have calculated values of the critical disorder $W_{c}$, the critical exponent $\nu$, and the mass exponent $\tau$. Only two sizes of systems have been analyzed in this work. Since considerable computing time is saved by this, we treated very large systems $\left(L_{\max }=256\right)$. To our knowledge, this is the largest scaling analysis in numerical works for twodimensional symplectic systems. Therefore, the obtained values of $W_{c}=5.86 \pm 0.04, \nu=2.41 \pm 0.24$, and $\tau(2)=1.62$ \pm 0.01 are quite reliable.

\section{ACKNOWLEDGMENTS}

We are grateful for valuable discussions with T. Nakayama, T. Hokari, and T. Ohtsuki. Numerical calculations were performed on the FACOM VPP 500 of the Supercomputer Center, Institute for Solid State Physics, University of Tokyo. This work was supported in part by a Grant-in-Aid for Scientific Research from the Ministry of Education, Science, and Culture of Japan.
*Present address: NTT Multimedia Service Promotion Headquarters, Chiyoda-ku, Tokyo 104-0004, Japan.

${ }^{1}$ See, for example, B. Kramer and A. MacKinnon, Rep. Prog. Phys. 56, 1469 (1993).

${ }^{2}$ D. C. Licciardello and D. J. Thouless, J. Phys. C 11, 925 (1978).

${ }^{3}$ A. MacKinnon and B. Kramer, Phys. Rev. Lett. 47, 1546 (1981); Z. Phys. B 53, 1 (1983).

${ }^{4}$ Y. Huo and R. N. Bhatt, Phys. Rev. Lett. 68, 1375 (1992).

${ }^{5}$ B. I. Shklovskii, B. Shapiro, B. R. Sears, P. Lambrianides, and H. B. Shore, Phys. Rev. B 47, 11487 (1993).

${ }^{6}$ E. Hofstetter and M. Schreiber, Phys. Rev. B 49, 14726 (1994).

${ }^{7}$ K. Yakubo and Y. Goto, Phys. Rev. B 54, 13432 (1996).

${ }^{8}$ F. Wegner, Z. Phys. B 36, 209 (1980).

${ }^{9}$ H. Aoki, J. Phys. C 16, L205 (1983).

${ }^{10}$ S. Hikami, A. Larkin, and Y. Nagaosa, Prog. Theor. Phys. 63, 707 (1980).

${ }^{11}$ K. B. Efetov, Adv. Phys. 32, 53 (1983).

${ }^{12}$ S. V. Kravchenko, W. E. Mason, G. E. Bowker, J. E. Furneaux, V. M. Pudalov, and M. D'Iorio, Phys. Rev. B 51, 7038 (1995); S. V. Kravchenko, D. Simonian, M. P. Sarachik, W. Mason, and J. E. Furneaux, Phys. Rev. Lett. 77, 4938 (1996).

${ }^{13}$ Y. Hanein, U. Meirav, D. Shahar, C. C. Li, D. C. Tsui, and Hadas Shtrikman, Phys. Rev. Lett. 80, 1288 (1998).

${ }^{14}$ M. Y. Simmons, A. R. Hamilton, M. Pepper, E. H. Linfield, P. D. Rose, D. A. Ritchie, A. K. Savchenko, and T. G. Griffiths, Phys. Rev. Lett. 80, 1292 (1998).
${ }^{15}$ For a brief review, see M. Schreiber and H. Grussbach, Mod. Phys. Lett. B 6, 851 (1992).

${ }^{16}$ V. I. Fal'ko and K. B. Efetov, Europhys. Lett. 32, 627 (1995).

${ }^{17}$ D. Stauffer and A. Aharony, Introduction to Percolation Theory (Taylor \& Francis, London, 1992).

${ }^{18}$ G. Paladin and A. Vulpiani, Phys. Rep. 156, 147 (1987).

${ }^{19}$ S. Yoshino and M. Okazaki, J. Phys. Soc. Jpn. 43, 415 (1977).

${ }^{20}$ A. MacKinnon, in Localization, Interaction, and Transport Phenomena, edited by B. Kramer, G. Bergmann, and Y. Bruynseraede (Springer, Berlin, 1984), p. 90.

${ }^{21}$ S. N. Evangelou and T. Ziman, J. Phys. C 20, L235 (1987).

${ }^{22}$ T. Ando, Phys. Rev. B 40, 5325 (1989).

${ }^{23}$ R. Merkt, M. Janssen, and B. Huckestein, cond-mat/9803342.

${ }^{24}$ K. Yakubo, T. Nakayama, and H. J. Maris, J. Phys. Soc. Jpn. 60, 3249 (1991).

${ }^{25}$ W. H. Press, S. A. Teukolsky, W. T. Vetterling, and B. P. Flannery, Numerical Recipes in Fortran 77 (Cambridge University Press, Cambridge, 1992), Chap. 15.

${ }^{26}$ K. Slevin and T. Ohtsuki, Phys. Rev. Lett. 78, 4083 (1997).

${ }^{27}$ U. Fastenrath, G. Adams, R. Bundschuh, T. Hermes, B. Raab, I. Schlosser, T. Wehner, and T. Wichmann, Physica A 172, 302 (1991); U. Fastenrath, Helv. Phys. Acta 65, 425 (1992).

${ }^{28}$ S. N. Evangelou, Phys. Rev. Lett. 75, 2550 (1995).

${ }^{29}$ T. Kawarabayashi and T. Ohtsuki, Phys. Rev. B 53, 6975 (1996).

${ }^{30}$ L. Schweitzer and I. Kh. Zharekeshev, J. Phys.: Condens. Matter 9, L441 (1997). 